\title{
The genetic evidence for human origin of Jivaroan shrunken heads in collections from the Polish museums
}

\author{
Danuta Piniewska $^{1}$ [D $\cdot$ Marek Sanak $^{2} \cdot$ Marta Wojtas $^{1} \cdot$ Nina Polanska $^{1}$
}

Received: 2 August 2016/Accepted: 29 August 2016/Published online: 18 September 2016

(C) Springer-Verlag Berlin Heidelberg 2016. This article is published with open access at Springerlink.com

\begin{abstract}
Advances in forensic identification using molecular genetics are helpful in resolving some historical mysteries. The aim of this study was to confirm the authenticity of shrunken-head artifacts exhibited by two Polish museums. Shrunken heads, known as tsantsas, were headhunting trophies of South American Indians (Jivaroan). A special preparation preserved their hair and facial appearance. However, it was quite common to offer counterfeit shrunken heads of sloths or monkeys to collectors of curiosities. We sampled small skin specimens of four shrunken-head skin from the museum collection from Warsaw and Krakow, Poland. Following genomic DNA isolation, highly polymorphic short tandem repeats were genotyped using a commercial chemistry and DNA sequencing analyzer. Haplogroups of human Y chromosome were identified. We obtained an informative genetic profile of genomic short tandem repeats from all the samples of shrunken heads. Moreover, amplification of amelogenin loci allowed for sex determination. All four studied shrunken heads were of human origin. In two ones, a shared Y-chromosome haplogroup Q characteristic for Indigenous Americans was detected. Another artifact was counterfeited because Y-chromosome haplogroup I2 was found, characteristic for the Southeastern European origin. Commercial genetic methods of identification can be applied
\end{abstract}

Danuta Piniewska

danuta.piniewska@uj.edu.pl

1 Present address: Department of Forensic Medicine, Jagiellonian University Medical College, Grzegorzecka Str. 16, 31-531 Krakow, Poland

2 Department of Internal Medicine, Jagiellonian University Medical College, Krakow, Poland successfully in studies on the origin and authenticity of some unusual collection items.

Keywords Tsantsa/shrunken head · Short tandem repeats · Y-chromosome haplotype $\cdot$ Forensic identification

\section{Introduction}

The Jivaroan are known as Amazonian groups of indigenous peoples living in the headwaters of the Marañon River and its tributaries, in the mountainous region of northern Peru and eastern Ecuador. They are the second largest and one of the most studied Amazonian groups with a notable history of survival and defense against outsiders. The Jivaroan represent a small linguistic family consisting of dialects: Shuar, Achuar, Aguaruna, and Huambisa. All of them share similar customs and they are genetically related $[1,2]$.

In the nineteenth century, the Jivaroan peoples became famous among Europeans and Euro-Americans travelers for their elaborate process of taking and shrinking an enemy's head to make a tsantsa. Although headhunting has occurred in many regions of the world, the practice of shrinking human heads has only been documented in the northwestern region of the Amazon rainforest, performed by the Jivaroan peoples. The reason for that had a spiritual origin rather than to keep them as a trophy. According to Jivaroan beliefs, a vengeful soul or muisak of a victim had to be trapped inside the tsantsa to protect the killer against the revenge. This practice also prevented the muisak from entering the afterlife potentially harmful for the family of the murderer or could be useful to enhance yields of crops [1,2].

Tsantsas were made from men, women, and children. Steps in the process of shrinking the heads had often to be carried in camps along the way back home after the attack. The head 
was severed by cutting the skin at the extreme base of the neck, in a "V" shape just above the clavicles. The skull was often submerged in a river to ease separation of muscles and tendons connecting the skin to the skull. The skin was severed from bones using a knife and eyes were removed. The skin of the head with the hair attached was sunk into the boiling water for a period between $30 \mathrm{~min}$ to $2 \mathrm{~h}$ three times and then placed on a spear to dry. Boiling led to a significant contraction of the skin to about one third of the original size. Then, the mouth and eyelids were sewn shut and nostrils were sometimes plugged with a cotton or a pitch. Next, hot small pebbles and sand were filled into the interior of head to complete the making of the shrunken head. Ultimately, the head was dangled over a fire to darken and toughen the skin. Alternatively a charcoal ash was rubbed over the skin and a hot blade was pressed to the lips to dry them. After these steps generally taking from 2 to 3 days, a human head was reduced in size to about one fourth, roughly to the size of a fist. During this process, the hair was not reduced, lending a characteristic look of the small head with long hair [1-3]. The spiritual system underlying the manner in which these trophies were made and celebrated changed during the second half of the nineteenth century when the Europeans arrived in the area. Tsantsas became used as a currency of trade. This created an economic demand for shrunken heads and superseded the spiritual and practical reasons for headhunting. The rate of killings rose in order to supply collectors and tourists with tsantsas, which resulted in many tensions within the Jivaro world [4]. Nowadays, shrinking-of-heads practices are prohibited.

Requests for shrunken heads also encouraged other people, non-Jivaroan, to produce counterfeited tsantsas for purposes other than those originally intended. The heads were usually taken from sloths and monkeys or even from human morgue corpses $[4,5]$. After the World War II, two shrunken heads were found at the Buchenwald concentration camp and were among evidences in Nuremberg Nazi Trials because they were thought to be taken form prisoners [6]. Even the Jivaroan peoples sometimes made a tsantsa of a sloth's head as they believed that it was the only animal in the forest that had a vengeful soul. It served as a remedy for a warrior who successfully killed the enemy but failed to take his head. Sometimes, it was also a part of a passage ceremony by boys to enter their manhood [3].

Nowadays, it is estimated that up to $80 \%$ of shrunken heads found in museums or in private collections are counterfeits. Very few fake tsantsas were ever made closely following the Jivaroan methods [7]. An audit conducted in the 1990s at the Smithsonian's collection revealed that only five of 21 examined were authentic tsantsas made by the Jivaroan [8]. Previous studies on the shrunken heads were based on the macroscopic examination of the head and microscopic analysis of hairs and skin to determine their origin [3, 4, 9-11]. This examination was usually sufficient for confirmation of authenticity because forgers did not concern about traditional Jivaroan methods of preparation. They wanted the head to look as lifelike as possible to please the buyers. Despite this, some counterfeiters could have produced tsantsas almost identical to those made by the Jivaroan peoples and anatomy or microscopic analysis could fail [4]. Molecular genetics used in forensic medicine offer an advantage of authentication by DNA analysis, which is increasingly used by museums or private collectors $[5,10,12]$. The authenticity certificates are sometimes important because false or nonceremonial heads do not have to be a subject of eventual repatriation requested upon museum curators or private owners [13, 14]. The National Museum of the American Indian at the Smithsonian was the first one to repatriate tsantsas to the Shuar Federation in 1999 [15].

The aim of the current study was to confirm a human origin of the three shrunken heads preserved in the State Ethnographic Museum in Warsaw (no.1- PME 5261, no.2PME 12272, no.3- PME 5260) and one in the collection of the Museum of the Department of Forensic Medicine at Jagiellonian University Medical College in Krakow (no.4), Poland (Fig. 1).

The tsantsa from the State Ethnographic Museum in Warsaw originated from Ecuador (no.3- PME 5260 and no.1- PME 5261) and from Peru (no.2- PME 12272). The first two were purchased from Mr. Vladimir Orda in 1950. The third was purchased by Mr. Stanislaw Jamka, who acquired it from the Indians in exchange for the rifle and beads in the year 1934. The first notice about the exhibit no. 4 in the inventory of the Department of Forensic Medicine appeared in the year 1903. It is known that experiments on tsantsa preparation were conducted at the local autopsy room in the 1970-1980s of the nineteenth century, when Ludwig Teichmann was the head of the Department of Forensic Medicine in Krakow. This shrunken-head hair was cut to make them match the head size.

\section{Material and methods}

Genetic analysis was carried out in the DNA laboratory certified for forensic genetics and with respect to the all precautions to avoid contamination with other human DNA. Briefly, laboratory steps of identification were performed in separate rooms using laminar-flow hoods, sterile disposable plasticware, and reagents. Pipettor tips with filters were used for all liquid handling. Negative PCR controls of deionized water were used throughout the whole process.

The external layer of the skin was cleaned several times with $99.8 \%$ ethanol and flat cut using disposable and sterile scalpel blade. Then, samples $\left(\sim 0.5 \mathrm{~cm}^{2}\right.$ and $0.1 \mathrm{~cm}$ thick) were taken from the inner surface of the 


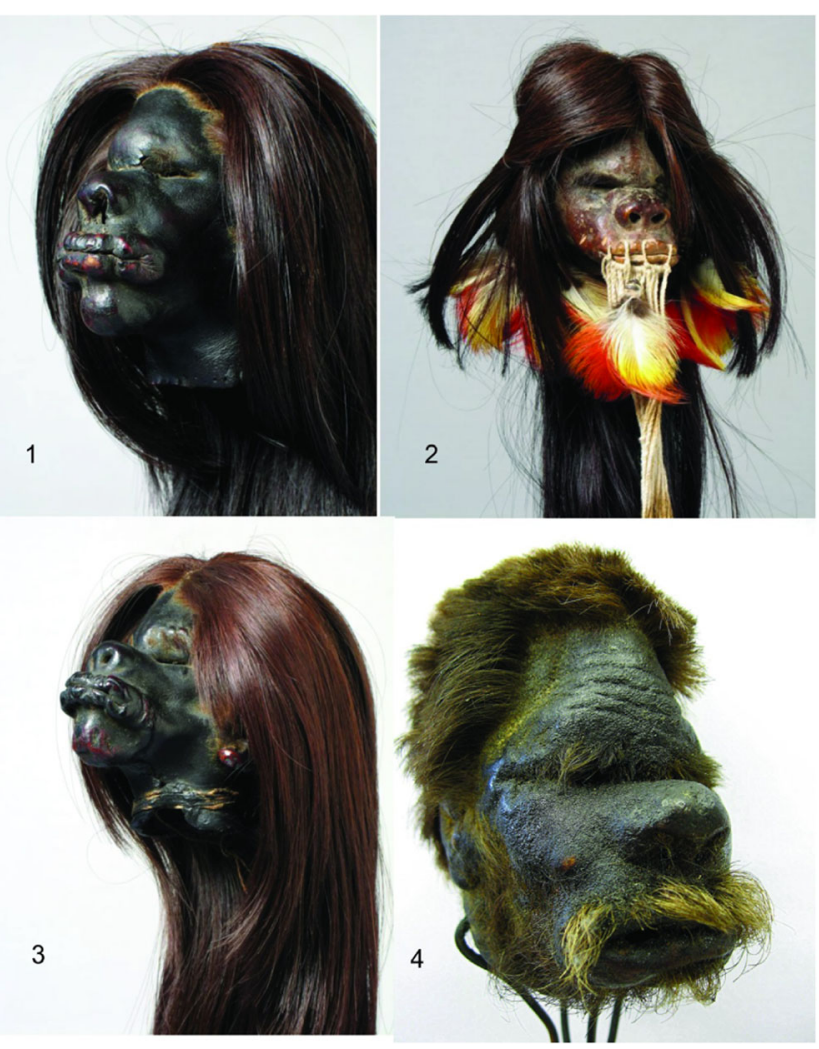

Fig. 1 View of the shrunken heads (The State Ethnographic Museum, Warsaw, Poland: no.1- PME 5261, no.2- PME 12272, no.3- PME 5260; The Museum of the Department of Forensic Medicine at Jagiellonian University Medical College, Krakow, Poland: no.4)

neck in all four shrunken heads. This surface had no direct contact with the outside environment. Each sample was collected into a sterile $1.5-\mathrm{mL}$ Eppendorf tube and incubated overnight at $42{ }^{\circ} \mathrm{C}$ prior to DNA extraction. DNA was extracted by enzymatic digestion and ionexchange column extraction using Sherlock $A X$ kit (A\&A Biotechnology, Gdynia, Poland) according to the manufacturer's recommendations. The concentration of DNA was determined by spectrophotometry at $260 \mathrm{~nm}$ using a GeneQuant RNA/DNA Calculator (Pharmacia Biotech, Cambridge, UK). PCR amplification was performed in a standard thermocycler (Perkin Elmer GeneAmp 2400, Applied Biosystems, Foster City, USA) and contained $4 \mathrm{ng}$ template DNA in a final reaction volume of $25 \mu \mathrm{L}$. Five commercially available reagent kits for typing of genomic short tandem repeat (STR) markers were used to study nuclear DNA (nDNA): AmpFISTR Identifiler PCR Amplification Kit, GlobalFiler Amplification Kit, AmpFISTR YFiler PCR Amplification Kit, and AmpFlSTR YFiler Plus PCR Amplification Kit (all from Applied Biosystems, Foster City, USA). Investigator Argus X-12 Kit was used to type for Xlinked STR (Qiagen GmbH, Hilden, Germany). These reagents were validated by the manufacturer and had appropriate genotypic controls and markers allelic ladders. The amplification products were analyzed using the AB3500 Genetic Analyser (Applied Biosystems, Foster City, USA). Fragment size and allele designation were determined by comparison with allelic ladders. The analytical threshold used for peak detection was set on $100 \mathrm{RFU}$ due to the high degradation of genetic material. Results were analyzed using GeneMapper ID-X Software (Applied Biosystems, Foster City, USA).

Minisequencing was performed for the Y-Chromosomal SNP marker M3 (rs38940) and M438 (rs17307294) in the male samples. Primer sequences used for amplification and sequencing were as follows: M3F-GGTACATTCGCGGG ATAA; M3R-GAATCTGAAATTTAAGGGCATC and M438F-GTGGCTGCATAAGGTAGATAAT; M438RAATTCTCTGATGGCGAGTC. Products of amplification were separated on agarose gel (M3-144 bp; M438 $237 \mathrm{bp}$ ) and extracted, next sequenced using BigDye Terminator v3.1 Cycle Sequencing Kit according to the manufacturer protocol and AB 3500 Genetic Analyser. Sequence analysis was performed with Sequencing Analysis Software v3.7 (Applied Biosystems, Foster City, USA).

Comparisons of Y-STR haplotypes were carried out against YHRD database release 51 (Y-Chromosome Haplotype Reference Database; available at http://www.yhrd.org). Haplogroup predictor software was used for a haplogroup ascertainment (www.hprg.com/hapest5/). The family relationship between tentatively consanguineous samples (no.1, no.2, no.3) was tested using DNA-View software v. 33.17 (Brenner C, Iowa City, USA).

Hair was collected from the tested exhibits by pulling them out with tweezers and inspected under a light microscope (Zeiss, Axioscope; Jena, Germany).

\section{Results}

The examined exhibits were genotyped for 21 autosomal STR markers (Table 1). Each of the tested samples revealed an incomplete autosomal STR profile. Failures to genotype were more common for the reaction products which size exceeded $250 \mathrm{bp}$. Amelogenin amplification products determined human sex in all four samples. Three of four tsantsas were males and one was a female. No amplification products have been ever observed in multiple blank extraction and PCR controls. The use of two different kits for the amplification of autosomal (Identifiler/GlobaFiler) and Y-STRs (Yfiler/Yfiler Plus) markers allowed to crosscheck the reliability of the results. Autosomal alleles almost completely overlapped for the both kits, whereas in two markers (TPOX, D18S51), better results were obtained using Identifiler. Analysis of Y-STR profile showed better amplification using Yfiler Plus kit. 
Table 1 The genetic profiles of the shrunken heads. Autosomal STR and X or Y amelogenin alleles

\begin{tabular}{|c|c|c|c|c|}
\hline STR loci & Shrunken head no.1 & Shrunken head no.2 & Shrunken head no.3 & Shrunken head no. 4 \\
\hline D3S1358 & 15 & 17 & $15-16$ & $17-18$ \\
\hline vWA & 16 & $15-19$ & 14 & $15-19$ \\
\hline D16S539 & $10-11$ & 10 & $10-12$ & - \\
\hline CSF1PO & - & $10-12$ & 12 & - \\
\hline TPOX & $8-11$ & $11-12$ & 12 & 8 \\
\hline Y indel & 2 & - & 2 & 2 \\
\hline Amelogenin & $\mathrm{XY}$ & $\mathrm{X}$ & $\mathrm{XY}$ & $\mathrm{XY}$ \\
\hline D8S1179 & $11-14$ & $10-12$ & $11-13$ & $13-15$ \\
\hline D21S11 & $31.2-33.2$ & 31.2 & $29-33.2$ & $30.2-32.2$ \\
\hline D18S51 & $14-18$ & $14-15$ & $14-15$ & $13-14$ \\
\hline DYS391 & - & - & 10 & - \\
\hline D2S441 & $10-11$ & $10-11$ & $11-16$ & $10-11$ \\
\hline D19S433 & $13-15$ & 14.2 & $14.2-15$ & $13.2-14$ \\
\hline TH01 & $7-9.3$ & $6-7$ & $7-9.3$ & $6-8$ \\
\hline FGA & $22-25$ & $21-24$ & $24-25$ & $20-21$ \\
\hline D22S1045 & $15-16$ & 16 & $15-16$ & $11-16$ \\
\hline D5S818 & 11 & $9-11$ & $11-13$ & $9-12$ \\
\hline D13S317 & 9 & $9-10$ & 9 & $12-13$ \\
\hline D7S820 & $12-14$ & $10-11$ & 10 & - \\
\hline SE33 & $17-26.2$ & $18-27.2$ & $26.2-29.2$ & - \\
\hline D10S1248 & 14 & $14-15$ & $9-14$ & $13-16$ \\
\hline D1S1656 & $15-17.3$ & $13-17$ & $14-15$ & 15 \\
\hline D12S391 & $19-20$ & 19 & $18-19$ & $17.3-18$ \\
\hline D2S1338 & $18-20$ & $18-20$ & $17-20$ & - \\
\hline
\end{tabular}

The genetic analysis showed progressive degradation a template quality with the amplicon size in all tested samples. The peak heights decreased with their migration time in the electropherogram and some allelic or locus dropouts were noticed.

The results of autosomal STR profiles from investigated tsantsa were compared to the only available profile obtained from the shrunken head displayed at the "Eretz Israel Museums, Tel-Aviv" and were found to be different [12].

The Y-chromosomal haplotype of the three male shrunken heads was obtained for 25 Y-STR markers (Table 2). Only the DYS533 marker did not reveal alleles for two samples (no.1, no.4). Interestingly, the two of the three individuals shared identical haplotype (no.1, no.3). In a search for geographic origin of the artifacts, we compared Y-STR haplotypes with published data on ethnic groups from Ecuador [16-19]. None of the obtained haplotypes had been previously observed among inhabitants of Ecuador. However, the modern ethnic composition in Ecuador is rather admixed. Mestizos are the largest population group (mixed descendant of indigenous Amerindians and Spanish colonists), who constitute just over $65 \%$; others are Indians (25\%), Europeans (7\%), and AfroEcuadorians (3\%) [16]. Noteworthy, the Y haplotype of the shrunken heads differed from the Waorani, the last semi- nomadic population of hunter-gatherer horticulturalists living in the Amazon region of Ecuador [17, 20].

The Y-Chromosome Haplotype Reference Database (YHRD; http://www.yhrd.org), was searched to answer whether two different haplotypes found in the examined shrunken heads existed in other populations. We found no entry among 6872 Y-STR haplotypes reported in this database. We also searched the most frequent minimal haplotypes within the YHRD database (i.e., DYS19-389I-389II-390-391392-393-385). One match in 160,693 worldwide reported haplotypes was found for sample no.1 and no.3 in Ecuador population, whereas five matches were observed for sample no.4, including two hits in the Eurasian-European-Eastern European populations (Hungary, Slovakia, Macedonia).

Using comparisons provided by Haplogroup Predictor (http://www.hprg.com/hapest5/) based on 22 Y-STR markers, $100 \%$ probability of Q haplogroup was obtained for sample no. 1 and no.3. These findings were reinforced by previous observations that all Waorani males belonged to haplogroup Q. This is the major lineage among the Native Americans, with Q-M3 (Q1a3a) being almost completely restricted to the Americas [20, 21]. A recent publication on the Waorani stated that more than $90 \%$ of the males belonged to a sub-haplogroup Q1a3a [22]. For 
Table 2 The genetic profiles of the shrunken heads. Ychromosomal STR alleles

\begin{tabular}{|c|c|c|c|}
\hline Y-STR loci & Shrunken head no.1 & Shrunken head no.3 & Shrunken head no. 4 \\
\hline DYS576 & 18 & 18 & 17 \\
\hline DYS389I & 14 & 14 & 12 \\
\hline DYS635 & 22 & 22 & 21 \\
\hline DYS389II & 32 & 32 & 30 \\
\hline DYS627 & 20 & 20 & 19 \\
\hline DYS460 & 10 & 10 & 10 \\
\hline DYS458 & 19 & 19 & 17 \\
\hline DYS19 & 12 & 12 & 16 \\
\hline YGATAH4 & 11 & 11 & 11 \\
\hline DYS448 & 20 & 20 & 19 \\
\hline DYS391 & 10 & 10 & 10 \\
\hline DYS456 & 17 & 17 & 15 \\
\hline DYS390 & 23 & 23 & 24 \\
\hline DYS438 & 11 & 11 & 10 \\
\hline DYS392 & 14 & 14 & 11 \\
\hline DYS518 & 41 & 41 & 40 \\
\hline DYS570 & 18 & 18 & 18 \\
\hline DYS437 & 14 & 14 & 15 \\
\hline DYS385 & $14-17$ & $14-17$ & $14-15$ \\
\hline DYS449 & 31 & 31 & 29 \\
\hline DYS393 & 14 & 14 & 13 \\
\hline DYS439 & 12 & 12 & 12 \\
\hline DYS481 & 24 & 24 & 30 \\
\hline DYF387S1 & $35-41$ & $35-41$ & $37-39$ \\
\hline DYS533 & - & 11 & - \\
\hline
\end{tabular}

the sample no.4, probability of I2a haplogroup was also $100 \%$. The haplogroup I represents one of two major European Y chromosome haplogroups [23-25] and by origin is absent elsewhere. Subclade I1 is the most frequent in the Northern Europe, whereas subclade 12 is the most frequent haplogroup in the Eastern Europe and the Balkans [24]. This coincides with the origin of the head and the reported history of its preparation. A contemporary contamination of this exhibit with a single individual DNA could not be excluded. However, a similar pattern of nuclear DNA template degradation in all samples and no traces of any other profile admixture advocates against pre-laboratory contamination.

Additionally, in order to confirm the origin of the tsansta haplotype, minisequencing was performed for the YChromosomal SNP markers: M3 for Q1a3a haplogroup and M438 for I2 haplogroup. These results confirmed the predicted haplogroup Q1a3a of native South Americans for tsantsa no. 1 and no.3 (rs3894C > T variant).

We also investigated X-chromosome markers. Out of the four shrunken heads, only three were successfully typed for 12 X-STR's markers (Table 3), all from the
State Ethnographic Museum in Warsaw (no.1, no.2, no.3). The samples no.1 and no.2 revealed a complete X-STR profile; a partial one was obtained for the sample no.3. Genotyping of the sample no.4 ascertained partial genotype of four X-STR markers, and this failure could result from partial degradation of DNA stored frozen for a few months before the assay. All the samples had different X-STR profiles and the subjects were not closely related through the maternal line. We compared these data to the ethnic groups from Ecuador [20, 26, 27]. None of the resulting profiles had been previously observed in these populations.

To estimate the probability of kinship between the tsantsa from the State Ethnographic Museum, especially heads no.1 and no.3, we performed statistical calculations based on the autosomal markers using automatic kinship module of DNA-View software. There was no evidence for familial relationships between the individuals no.1, no.2, and no.3, based on the population of Amerindian Kichwas [28], Ecuador [29], and Peru [30]. The probabilities were less than $0.3(\mathrm{LR}=0.03)$ for the hypothesis that the heads no.2 and no.3 are biological 
Table 3 The genetic profiles of the shrunken heads. Xchromosomal STR alleles

\begin{tabular}{lllll}
\hline X-STR loci & Shrunken head no.1 & Shrunken head no.2 & Shrunken head no.3 & Shrunken head no.4 \\
\hline AMEL & XY & XX & XY & XY \\
DXS10148 & 24.1 & 27.1 & 26.1 & - \\
DXS10135 & 15 & 24 & 20 & - \\
DXS8378 & 10 & 10 & 10 & 9 \\
DXS7132 & 14 & $13-15$ & - & - \\
DXS10079 & 22 & $20-25$ & - & - \\
DXS10074 & 15 & $15-18$ & 18 & 8 \\
DXS10103 & 19 & 16 & 19 & 20 \\
HPRTB & 14 & $13-15$ & 14 & - \\
DXS10101 & 30.2 & 33 & 30.2 & - \\
DXS10146 & 28 & $25-28$ & 27 & - \\
DXS10134 & 38 & 39 & 34 & - \\
DXS7423 & 15 & $14-15$ & 17 & \\
\hline
\end{tabular}

siblings. Even lower values were obtained for the heads no.1 and no.3. A hypothesis that they were half-siblings with a common father was not confirmed ( $\mathrm{LR}=1.074)$. However, using Brazilian Amazon Region population frequencies [31], individuals no.1 and no.3 had 0.99053 probability $(\mathrm{LR}=104.6)$ of having a common father/male ancestor, which could explain their identical Y-STR profile. Assuming that they were biological siblings, probability was $0.8590(\mathrm{LR}=6.092)$ using the Brazilian Amazon Region data while for the remaining population data LR value dropped below 0.5 .

Hair samples from tested exhibits studied under microscope (Fig. 2) had no hair roots visible. Apparently, this was not possible to obtain the roots while collecting samples from the hard and shrunk scalp. It is also plausible that the process of preparation (boiling in water and drying over fire) could destruct hair roots. The hair medulla was less than one-third width of the shaft, amorphous, and mostly discontinuous (Fig. 2). These observations were compatible with human hair histology.

\section{Conclusions}

Analysis of nuclear short tandem repeats located at autosomal or sex chromosomes proved that all the studied shrunken heads were of human origin. Nevertheless, YSTR haplogroup I2 of the sample no.4 suggested a Southeastern European ancestry precluding a genuine Jivaroan origin. Two other samples (no.1, no.3) were Amerindians and probably consanguineous by a common male ancestor, because they shared identical profile of Ychromosome haplogroup Q1a2-M3. This haplogroup is characteristic for the Native Americans (Ecuador).

Calculations using automatic kinship module of DNAView software did not provide any strong evidence of familial relationships between the individuals no. 1 or no. 2 and no. 3 using autosomal STR but it was plausible that individuals no. 1 and no.3 had likely (LR=104.6) a common male ancestor. They also shared identical Y-STR haplotype.

Certification of tsantsa authenticity by macroscopic or microscopic analyses sometimes is disputable. Preparation
Fig. 2 Microscopic view of hair samples from the shrunken heads (images at $\times 40$ magnification)

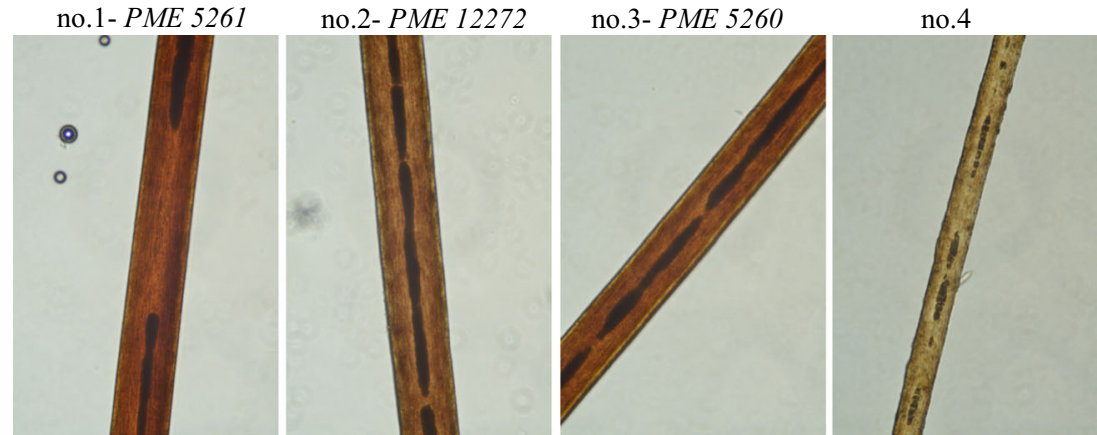


of some counterfeiters may employ a process very similar to the genuine tsantsa preparation by the Jivaroan peoples. On the other hand, some variants of the classic technique among authentic tsantsas also existed, or alternatively, some later modifications could have been done by a later owner. The genetic profiling together with traditional anthropological identification techniques seemed very helpful for determining the human origin of a shrunken head and their geographic provenance.

Acknowledgments We acknowledge cooperation of the State Ethnographic Museum in Warsaw providing skin samples of the shrunken heads for this study and the help of Adam Gross, M.D. Ph.D., who collected these samples. Mr. Edward Koprowski photographed the material in the State Ethnographic Museum in Warsaw.

\section{Compliance with ethical standards}

Disclosure The authors have approved the final article and are in agreement with its content.

Conflict of interests The authors declare that they have no conflict of interest.

Open Access This article is distributed under the terms of the Creative Commons Attribution 4.0 International License (http:// creativecommons.org/licenses/by/4.0/), which permits unrestricted use, distribution, and reproduction in any medium, provided you give appropriate credit to the original author(s) and the source, provide a link to the Creative Commons license, and indicate if changes were made.

\section{References}

1. Jandial R, Hughes SA, Aryan HE, Lawrence MF, Levy M (2004) The science of shrinking human heads: tribal warfare and revenge among the South American Jivaro-Shuar. Neurosurgery $55: 1215-1221$

2. Harner MJ (1984) The Jivaro: people of the sacred waterfalls, Secondth edn, New York: Natural History Press. University of California Press, Berkeley and Los Angeles

3. Charlier P, Huynh-Charlier I, Brun L, Herve C, de la Grandmaison GL (2012) Shrunken head (tsantsa): a complete forensic analysis procedure. Forensic Sci. Int. 222:399 (e1-5)

4. Mutter GL (1975) Jivaro tsantsas, authentic and forged: a study of two shrunken heads in the Mutter Museum. Trans Stud Coll Physicians Phila 43:78-82

5. Sauvageau A, Kremer C, Brochu V, Julien F, Racette S (2009) Jivaro tsantsas or shrunken head: an expertise of authenticity evaluation. Am J Forensic Med Pathol 30(1):72-74

6. Douglas L (1998) The shrunken head of Buchenwald: icons of atrocity at Nuremberg. Representations 63:39-64

7. Rubenstein S (2007) Circulation, accumulation and the power of Shuar shrunken heads. Cult Anthropol 22:357-399

8. Aufderheide AC (2003) The scientific study of mummies. Cambridge University Press, Cambridge, pp 93-96

9. Post P, Farrington D (2005) Histological and histochemical examination of American Indian scalps, mummies, and a shrunken head. Am J Phys Anthropol 30:269-294
10. Reichenpfader B, Buzina W, Roll P (2009) The mystery of shrinking heads. Forensic Sci Int Suppl 1:22-23

11. Houlton T (2012) Facial analysis of a Tsantsa (shrunken head). AXIS 4(1):1-20

12. Hermon D, Gafny R, Zamir A, Hadas L, Faerman M, Kahila BarGal G (2011) The genetic signature of a shrunken head. Archaeol Anthropol Sci 3:223-228

13. Rubenstein S (2004) Shuar migrants and shrunken heads face to face in a New York museum. Anthropol Today 20(3):15-18

14. Coughlan S (2007) Museum offered head for shrinking. BBC News, United Kingdom

15. Clark, J., (2011) How Shrunken Heads Work. HowStuffWorks.com [Online- access date 11 May 2011] Available at: http://people. howstuffworks.com/shrunken-head.htm

16. Baeza C, Guzmán R, Tirado M (2007) Population data for 15 Ychromosome STRs in a population sample from Quito (Ecuador). Forensic Sci Int 173:214-218

17. González-Andrade F, Roewer L, Willuweit S, Sánchez D, Martínez-Jarreta B (2009) Y-STR variation among ethnic groups from Ecuador: Mestizos, Kichwas, Afro-Ecuadorians and Waoranis. Forensic Sci Int Genet 3(3):83-91

18. González-Andrade F, Bolea M, Martínez-Jarreta B, Sánchez D (2006) Genetic analysis of autosomic and Y-chromosome STRPCR polymorphisms of the Huaoranis, the last nomad tribe in Ecuador. Int Congr Ser 1288:331-333

19. González-Andrade F, Sánchez D, Martínez-Jarreta B, Budowle B (2008) Y-chromosome STR haplotypes in three different population groups from Ecuador (South America). J Forensic Sci 53(2):512-514

20. Cardoso S, Alfonso-Sánchez MA, Valverde L, Sánchez D, Zarrabeitia MT, Odriozola A, Martínez-Jarreta B, de Pancorbo MM (2012) Genetic uniqueness of the Waorani tribe from the Ecuadorian Amazon. Heredity 108(6):609-615

21. Karafet TM, Mendez FL, Meilerman MB, Underhill PA, Zegura SL, Hammer MF (2008) New binary polymorphisms reshape and increase resolution of the human Y chromosomal haplogroup tree. Genome Res 18(5):830-838

22. Geppert M, Baeta M, Nunez C, Martınez-Jarreta B, Zweynert S, Cruz OW et al (2011) Hierarchical Y-SNP assay to study the hidden diversity and phylogenetic relationship of native populations in South America. Forensic Sci Int Genet 5:100-104

23. Jobling MA, Tyler-Smith C (2003) The human Y chromosome: an evolutionary marker comes of age. Nat Rev Genet 4:598-612

24. Rootsi S, Magri C, Kivisild T, Benuzzi G, Help H, Bermisheva M, Kutuev I, Barac L, Pericic M, Balanovsky O et al (2004) Phylogeography of Y-chromosome haplogroup I reveals distinct domains of prehistoric gene flow in Europe. Am J Hum Genet 75: $128-137$

25. Hammer MF, Karafet TM, Redd AJ, Jarjanazi H, SantachiaraBenerecetti S, Soodyall H, Zegura SL (2001) Hierarchical patterns of global human Y-chromosome diversity. Mol Biol Evol 18:1189-1203

26. Gaviria A, Zambrano AK, Morejón G, Aguirre V, Vela M, Builes JJ, Burgos G (2013) Genetic characterization of an XSTR decaplex on the population of Pichincha (Ecuador). Forensic Sci Int 4:144-145

27. Zambrano AK, Morejón G, Galarza J, Pastas V, Castillo G, Vela M, Gaviria A (2015) Genetic data of an X-STR decaplex in Ecuadorian population (P). Forensic Sci Int 5:91-92

28. González-Andrade F, Sánchez-Q D, Martínez-Jarreta B (2006) Genetic analysis of the Amerindian Kichwas and Afroamerican descendents populations from Ecuador characterised by 15 STRPCR polymorphisms. Forensic Sci Int 160(2-3):231-235

29. Gómez MA, Peñaherrera MS, Aguirre-Tello V, Vela-Cavinato M, Giovambattista G (2008) Allele frequencies of 15 STR loci 
in the population of the city of Quito, Ecuador. J Forensic Sci 53(2):510-511

30. Pérez L, Hau J, Izarra F, Ochoa O, Zubiate U, García O (2003) Allele frequencies for the 13 CODIS STR loci in Peru. Forensic Sci Int 132(2):164-165
31. Rodrigues EM, Palha Tde J, dos Santos SE (2007) Allele frequencies data and statistic parameters for 13 STR loci in a population of the Brazilian Amazon Region. Forensic Sci Int 168(2-3):244-247 